\title{
G s-costem \\ Risk Factors for the Morbidity and Prognosis of Lung Metastases in Newly Diagnosed Ovarian Carcinoma: A Large Population-based Study
}

\section{Shuning Ding}

The First School of Clinical Medicine, Zhejiang Chinese Medical University, Hangzhou 310053

\section{Kaibo Guo}

The First School of Clinical Medicine, Zhejiang Chinese Medical University, Hangzhou 310053

\section{Linqin Wu}

The First School of Clinical Medicine, Zhejiang Chinese Medical University, Hangzhou 310053

\section{Dongxu Li}

The Third School of Clinical Medicine (School of Rehabilitation Medicine), Zhejiang Chinese Medical University, Hangzhou 310053

\section{Peipei Wang}

The First School of Clinical Medicine, Zhejiang Chinese Medical University, Hangzhou 310053

\section{Leitao Sun}

Department of Medical Oncology, The First Affiliated Hospital of Zhejiang Chinese Medical University, Hangzhou 310006

\section{Shanming Ruan ( $\nabla$ shanmingruan@zcmu.edu.cn )}

Department of Medical Oncology, The First Affiliated Hospital of Zhejiang Chinese Medical University, Hangzhou 310006

\section{Fangfang Tao}

Department of Immunology and Microbiology, School of Basic Medical Sciences, Zhejiang Chinese Medical University, Hangzhou 310053

\section{Research Article}

Keywords: lung metastasis, ovarian cancer, nomogram, SEER

Posted Date: August 18th, 2021

DOI: https://doi.org/10.21203/rs.3.rs-737681/v1

License: (c) (i) This work is licensed under a Creative Commons Attribution 4.0 International License. Read Full License 


\section{Abstract}

Background: To evaluate the risk factors for the morbidity and prognosis of lung metastases (LM) in patients with newly diagnosed ovarian carcinoma (OC).

Methods: Based on the Surveillance, Epidemiology, and End Results (SEER) dataset, OC patients from 2010 and 2016 were retrospectively analyzed. Risk factors for the morbidity of LM in OC patients and their survival were assessed by logistic regression analysis and Kaplan-Meier and Gray method, respectively. Cox regression analysis was performed to identify risk factors for the prognosis of OC patients with $L M$, and their prognostic potentials were further validated by two established nomograms.

Results: There are 27,123 eligible OC patients were enrolled in the study, with the morbidity of LM at $5.61 \%(1,521 / 27,123)$. Logistic regression models illustrated that T3 stage [odds ratio $(O R)=2.74$, $95 \% \mathrm{Cl}=2.09-3.66, P<0.01]$, advanced $\mathrm{N}$ stage $(\mathrm{OR}=1.86,95 \% \mathrm{Cl}=1.62-2.14, P<0.01)$, and the prevalence of bone metastasis $(\mathrm{OR}=3.78,95 \% \mathrm{Cl}=2.79-5.11, P<0.01)$, brain metastasis $(\mathrm{OR}=4.67,95 \% \mathrm{Cl}=2.50-8.63$, $P<0.01)$ and liver metastasis $(\mathrm{OR}=3.60,95 \% \mathrm{Cl}=3.14-4.12, P<0.01)$ were all significantly correlated with the morbidity of LM in OC patients. Median survival for OC patients with LM was 11 months (interquartile range, 3 to 25 months). Cox regression analyses illustrated over 80 years of age [hazard ratio $(H R)=2.52$, $95 \% \mathrm{Cl}=2.33-2.72, P<0.01]$ and positive expression of cancer antigen $125(\mathrm{CA}-125, \mathrm{HR}=1.63,95 \% \mathrm{Cl}=1.47-$

$1.82, P<0.01)$ were significantly correlated with the high mortality of $L M$, while chemotherapy $(H R=0.62$, $95 \% \mathrm{Cl}=0.59-0.65, P<0.01)$ was significantly correlated with the low mortality. Two nomograms were established to examine the concordance index (C-index), calibration curves, the area under the curve (AUC), decision curve analyses (DCAs) and clinical impact curves (CICs), which validated the prognostic potentials of identified risk factors in OC patients with LM.

Conclusion: The population-based cohort study provides references for guiding clinical screening and individualized treatment of OC patients with LM.

\section{Introduction}

Ovarian carcinoma $(\mathrm{OC})$ is the most common cause of death in the female genital system. The American Cancer Society reported that there were 21,750 new cases of $O C$ and 13,940 deaths in 2020[1]. Approximately $60 \%$ of OC patients were determined at an advanced stage. Previous studies reported that the invasion of $\mathrm{OC}$ cells mainly depends on the hematogenous circulation and lymphatic channels[2], and pulmonary metastases in $\mathrm{OC}$ always are classified at a lower-level outcome that notably lead to a poor prognosis[3, 4]. As the second most common distant metastatic site, the morbidity of lung metastasis (LM) ranges from $6 \%$ to $16 \%[5-8]$. Although surgery is preferred as the curative treatment for metastatic malignancies, a large number of affected people connot be operated because of the strict indications[9]. OC patients with LM can also be beneficial from systemic treatments, including chemotherapy, radiotherapy, targeted therapy, and immunotherapy [10-12]. Although these treatments do prolong the progression free survival, most patients would ultimately suffer from relapse or resistance[13]. 
Meanwhile, the huge economic burden also challenges them. It is necessary to explore risk factors for the morbidity and prognosis of $\mathrm{OC}$ patients with $\mathrm{LM}$, thus enhancing the survival outcomes.

Through literature review, risk factors and survival estimates of OC patients with LM have not been extensively analyzed. Therefore, it is essential to construct predictive models for designing prophylactic treatments and attentive nursing care for $\mathrm{OC}$ patients at a high risk of LM. This study aims to investigate risk factors for the morbidity and prognosis of newly diagnosed OC patients with LM and validate them by establishing nomograms.

\section{Materials And Methods \\ 2.1 Patient selection}

We searched and downloaded OC patients' medical records from the SEER 18 registry dataset (http://seer.cancer.gov), which covered more than a third of the American population on cancer morbidity and survival records. A total of 27,123 patients diagnosed as $\mathrm{OC}$ based on the International Classification of Diseases for Oncology, $3^{\text {rd }}$ Edition (ICD-0-3) Hist/behavior, malignant from January 2010 to December 2016 were recruited in our study. The flow diagram of participant inclusion and exclusion was presented in Figure 1. The ethical approval was not requested in this study because clinical data of recruited OC patients were collected from the open-access and anonymized data in the public SEER dataset.

\subsection{Variable statement}

The demographic and clinical characteristics mentioned were identified and prescribed as following: year of diagnosis (2010-2012, 2013-2014, 2015-2016), age at diagnosis (18-49, 50-69, 70-79, $\geq 80$ years), race (black, white, other/not stated), marital status (married, unmarried, unknown), histology (non-serous, serous adenocarcinoma, other/not stated), grade (I=well differentiated, II=moderately differentiated, $\mathrm{III}=$ poorly differentiated, IV=undifferentiated, unknown), tumor size ( $<50 \mathrm{~mm}, 50-120 \mathrm{~mm}, \geq 120 \mathrm{~mm}$, not stated), examined regional nodes number $(0,1-10, \geq 11$, not stated), surgical treatments on primary site (unilateral, bilateral/other, no/unknown), tumor phase (T1, T2, T3, not stated), regional lymph node phase (N0, N1, not stated), CA-125 (negative, positive, borderline/unknown), the usage of radiotherapy and chemotherapy, as well as the appearance of bone metastasis, brain metastasis, and liver metastasis.

\subsection{Nomogram construction and validation}

In the cohort, the classified variables were expressed as the number and its percentage ( $\mathrm{N}, \%)$. Follow-up analyses were conducted to assess independent risks for the prognosis of OC with LM. Univariable and multivariable logistic regression models were introduced to identify risk factors for the morbidity of LM in newly diagnosed $\mathrm{OC}$ patients, and adjusted and unadjusted proportional hazard models were used to distinguish prognostic factors for OC with LM. Afterwards, two nomograms were established to quantify 
the prediction capacity. Based on risk scores of overall survival (OS) in the nomogram, patients were categorized into low-risk and high-risk subgroups. Differences between two subgroups were assessed by depicting the clinic effect curve. Furthermore, Kaplan-Meier survival curves were depicted to assess the overall survival of OC patients with LM. To avoid the impact of other critical illnesses, cancer-specific survival analyses were performed by the cumulative incidence function. Notably, the accuracy of nomogram was detected and validated by operating calibration plots. Also, DCAs and CICs were designed to calculate the net avails for each risk threshold probability.

\subsection{Statistical analysis}

R software (version 3.6.1, https://www.r-proje ct.org/) was employed for statistical analyses. The categorical data were measured by Fisher's exact test or Chi-square test. Nomograms based on regression models, calibration curves and survival-related curves were all drawn via diverse functional packages namely RMS, Foreign, Survival, Cmprsk and other software.

(https://www.mskcc.org/departments/epidemiologybiostatistics/health-outcomes/tutorial-r) A two-tailed $P$ value $<0.05$ was considered as statistical significance, $\left({ }^{*} P<0.05, * * P<0.01\right)$.

\section{Results}

\subsection{Baseline characteristics of OC patients}

As shown in Table 1, a total of 27,123 eligible OC patients were recruited and their mean age and median survival time were $60.39 \pm 14.90$ years and 22 months (interquartile range $9-43$ months), respectively. Among them, $5.61 \%(\mathrm{~N}=1,521)$ developed $\mathrm{LM}$ with the mean age and median survival time were $65.37 \pm 13.74$ years and 11 months (interquartile range $3-25$ months), respectively. Other demographic and medical traits of recruited OC patients were presented as well. The remarkable differences included the age, race, marital status, histology, grade, tumor size, the number of regional nodes examined, radiotherapy, chemotherapy, surgery scope, $\mathrm{T}$ stage, $\mathrm{N}$ stage, $\mathrm{CA}-125$, bone metastasis, brain metastasis and liver metastasis.

\subsection{Independent risk factors for the morbidity of LM in OC patients and nomogram establishment}

Unadjusted and adjusted logistic regression analyses were applied to assess independent risk factors for the morbidity of LM in newly diagnosed OC patients. It was shown that the histology, grade, the number of examined regional nodes number, treatment strategies like chemotherapy and surgery, $\mathrm{T}$ and $\mathrm{N}$ stage, CA-125, and the incidence of other distant metastases were correlated with the morbidity of LM in OC patients (Table 2). The morbidity of LM in OC patients with the histological subtype of serous was significantly lower than those with non-serous adenocarcinoma $(\mathrm{OR}=0.80,95 \% \mathrm{Cl}=0.70-0.92, P<0.01)$ was 
lower than those with non-serous adenocarcinoma. Concerning tumor grade, poorly differentiated $(\mathrm{OR}=2.71,95 \% \mathrm{Cl}=1.53-5.34, P<0.01)$, and undifferentiated $\mathrm{OC}$ patients $(\mathrm{OR}=2.84,95 \% \mathrm{Cl}=1.60-5.61$, $P<0.01)$ had a significantly higher risk of LM development than well differentiated ones. In addition, OC patients with more than 10 examined lymph nodes had a significantly lower risk for the morbidity of LM than those without lymph nodes detection $(\mathrm{OR}=0.41,95 \% \mathrm{Cl}=0.33-0.51, P<0.01)$. Advanced $\mathrm{T}$ and $\mathrm{N}$ stage, especially T3 stage $(\mathrm{OR}=2.74,95 \% \mathrm{Cl}=2.09-3.66, P<0.01)$ and $\mathrm{N} 1$ stage $(\mathrm{OR}=1.86,95 \% \mathrm{Cl}=1.62-2.14$, $P<0.01$ ) were risk factors for $\mathrm{LM}$ development in $\mathrm{OC}$ patients. Patients with surgical treatment of bilateral ovaries or more areas had a higher risk of LM development than those receiving unilateral ovary surgery $(\mathrm{OR}=1.30,95 \% \mathrm{Cl}=1.07-1.59, P<0.01)$, which might be attributed to disease development itself. Besides, radiotherapy $(\mathrm{OR}=1.19,95 \% \mathrm{Cl}=0.79-1.74, P<0.01)$, chemotherapy $(\mathrm{OR}=1.39,95 \% \mathrm{Cl}=1.20-1.62, P<0.01)$, bone metastasis $(\mathrm{OR}=3.78,95 \% \mathrm{Cl}=2.79-5.11, P<0.01)$, brain metastasis $(\mathrm{OR}=4.67,95 \% \mathrm{Cl}=2.50-8.63$, $P<0.01)$ and liver metastasis $(\mathrm{OR}=3.60,95 \% \mathrm{Cl}=3.14-4.12, P<0.01)$ were all risk factors for the morbidity of LM in OC patients.

Subsequently, we established a nomogram to intuitively display score assignments and predictive probability of the risk factors (Figure 2A). Simultaneously, the calibration curve with the C-index of 0.807 suggested an extremely consistency between actual observations and the probability of prediction (Figure 2B). DCAs and CICs illustrated that threshold probabilities at 0-0.3 were the most favorable predictor of LM in accordance with our nomogram model (Figure 2C-D).

\subsection{Survival analyses of OC patients with LM}

Kaplan-Meier method was adopted to detect the influence of LM on the outcome of OC patients. As shown in Figure 3A, OS curves revealed that $L M$ development was significantly correlated to the prognosis of $\mathrm{OC}(\mathrm{HR}=1.36,95 \% \mathrm{Cl}=1.27-1.45, P<0.01)$. The OS was significantly worse in $\mathrm{OC}$ patients with over 80 years of age (Figure 3B, $P<0.01$ ), poorly differentiated and undifferentiated neoplasm (Figure $3 \mathrm{C}$, $P<0.01$ ), bone metastasis (Figure 3D, $P<0.01$ ), brain metastasis (Figure $3 \mathrm{E}, P<0.01$ ) and liver metastasis (Figure $3 F, P<0.01$ ) than those of controls. Meanwhile, we found that LM was significant correlated with the major cause of death in $\mathrm{OC}$ patients rather than other diseases via gray method [sub-distribution hazard ratio $(\mathrm{SHR})=3.08,95 \% \mathrm{Cl}=2.89-3.28, P<0.01]$ (Figure $3 \mathrm{G})$.

\subsection{Prognostic factors for $\mathrm{OC}$ and nomogram establishment}

Prognostic factors for OC patients were analyzed using the cox regression model (Table 3). OC patients aging 50-69 years $(\mathrm{HR}=1.41,95 \% \mathrm{Cl}=1.32-1.50, P<0.01)$, aged $70-79(\mathrm{HR}=1.79,95 \% \mathrm{Cl}=1.67-1.93, P<0.01)$, and over 80 years $(\mathrm{HR}=2.52,95 \% \mathrm{Cl}=2.33-2.72, P<0.01)$ were detected to have a higher risk of death. Concerning treatment strategies, a lower risk of death was detected in $\mathrm{OC}$ patients treated with chemotherapy $(\mathrm{HR}=0.62,95 \% \mathrm{Cl}=0.59-0.65, P<0.01)$. $T$ stage was a significant risk factor for the prognosis of $\mathrm{OC}$, especially $\mathrm{T} 3$ stage $(\mathrm{HR}=3.66,95 \% \mathrm{Cl}=3.36-3.99, P<0.01)$. Likewise, regional lymph nodes metastases $(\mathrm{HR}=1.40,95 \% \mathrm{Cl}=1.33-1.47, P<0.01)$, positive $\mathrm{CA}-125(\mathrm{HR}=1.63,95 \% \mathrm{Cl}=1.47-1.82$, 
$P<0.01)$, bone metastasis $(\mathrm{HR}=1.58,95 \% \mathrm{Cl}=1.35-1.85, P<0.01)$, brain metastasis $(\mathrm{HR}=1.64,95 \% \mathrm{Cl}=1.21$ $2.21, P<0.01)$ and liver metastasis $(\mathrm{HR}=1.38,95 \% \mathrm{Cl}=1.30-1.47, P<0.01)$ were all independent risk factors for the prognosis of $\mathrm{OC}$.

According to the results of cox regression analysis, significant risk factors for the prognosis of OC were subjected to the establishment of a nomogram for determining the 3-year and 5-year survival rate (Figure 4A). Stratified by the medium scores from the nomogram, the clinic effect curve revealed that the high survival feasibility of low-risk subgroup was significantly superior to that of high-risk subgroup (Figure 5, $\mathrm{HR}=1.06,95 \% \mathrm{Cl}=1.03-1.10, P<0.01)$. Furthermore, the calculated 3-year and 5-year AUC (0.812 and 0.818, respectively, Figure 4B) and the solid lines closed to the diagonal lines (Figure 4C) both displayed the excellent accuracy of the prediction.

\section{Discussion}

Ovarian carcinoma is regarded as the first leading cause of mortality among gynecological malignancies due to the high rates of advanced stage and recurrence. is regarded as the first leading cause of mortality among gynecological malignancies due to the high rates of advanced stage and recurrence.

Although risk factors for the prognosis of metastatic $\mathrm{OC}$ have been widely explored, shortcomings exist. Previous studies have obtained hazard factors for the morbidity and prognosis of $\mathrm{OC}$ with distant metastases, but a visualized figure to predict the probability is lacked[14, 15]. Nomograms, as a novel form of data visualization, have been well concerned to improve the efficiency for clinical applications. Yuan et al.[16] once revealed that the advanced $\mathrm{T}$ and $\mathrm{N}$ stage, other distant metastases were risk factors for the morbidity of LM in OC patients, and treatment strategies like active surgery and chemotherapy served as protective factors. However, inclusion and exclusion criteria are inconsistent. Whether there are complications that may significantly influence the OS of recruited patients remain unclear. Although the prognostic survival rate was predicted, the results had little significance since not all significant risk factors have been introduced in the multivariate cox regression model. As a result, predicted results were not representative for the actual HR.

Therefore, we identified risk factors for the morbidity of LM and prognosis of OC patients with LM by logistic regression analyses and proportional hazard analyses, followed by a direct visualization of the probabilities for LM development in OC patients and their OS through establishing nomograms. CA-125 is a large membrane glycoprotein belonging to the wide mucin family. Thirty years after its discovery, CA125 is still recommended as a vital tumor marker for OC, which is detected for reflecting cancer cell residue or recurrence in $\mathrm{OC}$ patients after the first-line therapy[17]. It is proved that a rising serum CA-125 level within the normal range is strongly associated with the risk of recurrence and the survival of OC[18], suggesting that the fluctuated CA-125 level is valuable for predicting the prognosis of OC. In this study, we not only detected the influence of CA-125 on the prognosis of OC patients with LM, but also tried to eliminate the intervention from other major diseases by calculating the cancer-specific survival. Moreover, multiple methods were adopted to determine the availability of nomograms. 
According to the cohort analyses, $5.61 \%$ of the included OC patients were diagnosed with LM and the median survival was 11 months. We found that LM was more likely developed in OC patients with a high tumor grade, non-serous adenocarcinoma, the usage treatment of radiotherapy and chemotherapy, progressive $\mathrm{T}$ and $\mathrm{N}$ stage, and other organ metastases. Older age, poorly differentiated and undifferentiated grade, lack of regional lymph node examination, radiotherapy, elevated CA-125, advanced $\mathrm{T}$ and $\mathrm{N}$ phase and other site metastases were significantly correlated with the poor prognosis of OC with LM. To verify the precision of nomograms, the C-index, calibration plots and the value of AUC demonstrated the high agreement on predictions. Moreover, the clinic effect curve showed the discrimination ability of models. Obviously, the survival probability in low-risk subgroup was dramatically that in higher than high-risk OC patients, indicating that identifying risk factors was instructive and meaningful for guiding prophylactic clinical treatment and improving the prognosis of OC patients.

According to the previously established nomogram on the morbidity of LM in OC patients, the serous adenocarcinoma is considered as the most aggressive subtype[19]. Inconsistently, our results revealed that patients with non-serous carcinoma of $\mathrm{OC}$, including mucinous, endometrioid and other subtypes had a memorably higher probability to develop LM. A growing number of studies have reported smoking increases the risk for the development of non-serous carcinomas, especially mucinous tumors, but clear association with serous subtypes is scant[20,21]. Besides, it has been widely accepted that smoke exposure increases the numbers of lung metastases[22, 23], which might explain the inverse distribution in the study. A previous study suggested that higher tumor grade and T stage were crucial risk factors for the prognosis of gynecological cancer patients with distant metastases[24]. Not surprisingly, we obtained the similar result that undifferentiation and poor differentiation grade, worse $\mathrm{T}$ and $\mathrm{N}$ stages and lack of regional node examination were significantly correlated with the risk of OC with LM.

As for the OS nomogram, we revealed that the prognosis of youngest OC patients aging18-49 years was better than older ones, which was consistent with previous findings[25, 26]. It is reported that an elevated CA-125 level indicated an ineffective treatment[27]. Likewise, our study found that elevated CA-125 level resulted in worse survival outcomes, regarded as the effective determinant for the prognosis of $\mathrm{OC}$ with LM. Notably, the application of chemotherapy brought out an opposite effect in two nomograms. Current data demonstrated that chemotherapy resistance of OC cells would contribute to the recurrence and metastases[28]. On the contrary, evidences also supported that chemotherapy is feasible for partial cytoreduction and prolonged survival[29,30]. Undeniably, chemotherapy is conducive to improve clinical response and outcome[31,32]. For cancer patients with regional lymph node involvement, surgery and chemotherapy are positive factors for OS[33], which was also validated in our cox regression analyses. However, for patients who relapse or die from the disease, the assessment of risk factors and biomarkers at the cellular level for chemotherapy response should be highlighted in the future[34].

Our study still had several limitations. Firstly, this population-based retrospective investigation lacked some pivotal clinical data, such as the detailed assessment about pulmonary metastatic tumors and more information on individual treatments. Secondly, the obtained morbidity of LM might produce regional biases because the model was registered in the United States. Thirdly, our results were 
remarkably underestimated because a large number of OC patients having LM later have not been recorded. Last but not the least, these models constructed from SOR database did not go through verifications by external data and should be constantly modified based on the clinical practice.

\section{Conclusion}

The retrospective study represented the largest dataset for LM development in OC patients and provided valuable nomograms about epidemiological characteristics and prognosis of advanced OC. Moreover, our findings suggested a strong reliability through multiple statistic approaches of calibration and discrimination. Hence, they had the potential to guide clinical diagnosis and individual treatments of OC with LM. In the future, laboratory investigations and large sample prospective clinical trials are demanded to further evaluate the molecular characteristics and treatment decisions for OC patients with LM.

\section{Declarations}

\section{Acknowledgements}

Not applicable.

\section{Authors' contributions}

S.D. and K.G. designed and conducted experiments. L.W. and D.L. recorded and compiled the data. P.W. and L.S. wrote the manuscript. S.R. and F.T. organized, conceived, and supervised the research. The whole authors participated and approved the manuscript.

\section{Funding}

This work was supported by National Natural Science Foundation of China (FT, No. 82074391), Zhejiang Provincial Programme of TCM (No. 2019ZQ013).

\section{Availability of data and materials}

All the records generated for this research is available in the SEER dataset (https://seer.cancer.gov/about/overview.html).

\section{Ethics approval and consent to participate}

This study depended on publicly available de-identified data from the SEER dataset that did not involve the use of personally identifiable information or interaction with the included population. The informed 
consent from the SEER registered cases in this study was not necessary and the authors obtained Limited-Use Data Agreements from SEER. No trial registration was required.

\section{Consent for publication}

Not applicable.

\section{Competing interests}

The authors announced that the study was performed in the absence of any financial or commercial relationships which could be construed as a potential conflict of interest.

\section{References}

1. Siegel RL, Miller KD, Fuchs HE, Jemal A: Cancer Statistics, 2021. CA Cancer J Clin 2021, 71(1):7-33.

2. Romani C, Zizioli V, Silvestri M, Ardighieri L, Bugatti M, Corsini M, Todeschini P, Marchini S, D'Incalci M, Zanotti L et al: Low Expression of Claudin-7 as Potential Predictor of Distant Metastases in HighGrade Serous Ovarian Carcinoma Patients. Frontiers in oncology 2020, 10:1287.

3. Chen X, Chen L, Zhu H, Tao J: Risk factors and prognostic predictors for Cervical Cancer patients with lung metastasis. Journal of Cancer 2020, 11(20):5880-5889.

4. Li Y, Zhou Z, Liu D, Zhou M, Tan F, Liu W, Zhu H: Predictive and Prognostic Factors of Synchronous Colorectal Lung-Limited Metastasis. Gastroenterology research and practice 2020, 2020:6131485.

5. Gardner AB, Charo LM, Mann AK, Kapp DS, Eskander RN, Chan JK: Ovarian, uterine, and cervical cancer patients with distant metastases at diagnosis: most common locations and outcomes. Clinical \& experimental metastasis 2020, 37(1):107-113.

6. Dauplat J, Hacker NF, Nieberg RK, Berek JS, Rose TP, Sagae S: Distant metastases in epithelial ovarian carcinoma. Cancer 1987, 60(7):1561-1566.

7. Cormio G, Rossi C, Cazzolla A, Resta L, Loverro G, Greco P, Selvaggi L: Distant metastases in ovarian carcinoma. International journal of gynecological cancer: official journal of the International Gynecological Cancer Society 2003, 13(2):125-129.

8. Güth U, Huang DJ, Bauer G, Stieger M, Wight E, Singer G: Metastatic patterns at autopsy in patients with ovarian carcinoma. Cancer 2007, 110(6):1272-1280.

9. Xu Y, Bai L, Zhang L, Mao F, Shen-Tu Y: Analysis of the Choice of Operation Mode and Prognosis Factors of Patients with Tumors of the Lung Metastasis. Zhongguo fei ai za zhi = Chinese journal of lung cancer 2015, 18(4):206-211.

10. Fleming C, Rimner A, Foster A, Woo KM, Zhang Z, Wu AJ: Palliative efficacy and local control of conventional radiotherapy for lung metastases. Annals of palliative medicine 2017, 6(Suppl 1):S21s27. 
11. Loizzi V, Leone L, Camporeale A, Resta L, Selvaggi L, Cicinelli E, Cormio G: Neoadjuvant Chemotherapy in Advanced Ovarian Cancer: A Single-Institution Experience and a Review of the Literature. Oncology 2016, 91(4):211-216.

12. Ventriglia J, Paciolla I, Pisano C, Cecere SC, Di Napoli M, Tambaro R, Califano D, Losito S, Scognamiglio G, Setola SV et al: Immunotherapy in ovarian, endometrial and cervical cancer: State of the art and future perspectives. Cancer treatment reviews 2017, 59:109-116.

13. van Zyl B, Tang D, Bowden NA: Biomarkers of platinum resistance in ovarian cancer: what can we use to improve treatment. Endocrine-related cancer 2018, 25(5):R303-r318.

14. Zhao H, Xu F, Li J, Ni M, Wu X: A Population-Based Study on Liver Metastases in Women With Newly Diagnosed Ovarian Cancer. Frontiers in oncology 2020, 10:571671.

15. Zhang C, Guo X, Peltzer K, Ma W, Qi L, Zhang Y, Han X, Baklaushev VP, Yao Y, Wang G et al: The prevalence, associated factors for bone metastases development and prognosis in newly diagnosed ovarian cancer: a large population based real-world study. Journal of Cancer 2019, 10(14):31333139.

16. Yuan Y, Guo F, Wang R, Zhang Y, Bai G: Development and validation of a nomogram to predict synchronous lung metastases in patients with ovarian cancer: a large cohort study. Bioscience reports 2020, 40(11).

17. Bottoni P, Scatena R: The Role of CA 125 as Tumor Marker: Biochemical and Clinical Aspects. Advances in experimental medicine and biology 2015, 867:229-244.

18. Piatek S, Panek G, Lewandowski Z, Bidzinski M, Piatek D, Kosinski P, Wielgos M: Rising serum CA-125 levels within the normal range is strongly associated recurrence risk and survival of ovarian cancer. Journal of ovarian research 2020, 13(1):102.

19. Rojas V, Hirshfield KM, Ganesan S, Rodriguez-Rodriguez L: Molecular Characterization of Epithelial Ovarian Cancer: Implications for Diagnosis and Treatment. International journal of molecular sciences 2016, 17(12).

20. Wentzensen N, Poole EM, Trabert B, White E, Arslan AA, Patel AV, Setiawan VW, Visvanathan K, Weiderpass E, Adami HO et al: Ovarian Cancer Risk Factors by Histologic Subtype: An Analysis From the Ovarian Cancer Cohort Consortium. Journal of clinical oncology: official journal of the American Society of Clinical Oncology 2016, 34(24):2888-2898.

21. La Vecchia C: Ovarian cancer: epidemiology and risk factors. European journal of cancer prevention: the official journal of the European Cancer Prevention Organisation (ECP) 2017, 26(1):55-62.

22. Lu LM, Zavitz CC, Chen B, Kianpour S, Wan Y, Stämpfli MR: Cigarette smoke impairs NK celldependent tumor immune surveillance. Journal of immunology (Baltimore, Md: 1950) 2007, 178(2):936-943.

23. McKenzie E, Hwang MK, Chan S, Zhang L, Zaki P, Tsao M, Barnes E, Razvi Y, Drost L, Yee C et al: Predictors of dyspnea in patients with advanced cancer. Annals of palliative medicine 2018, 7(4):427-436. 
24. Zhang Y, Guo Y, Zhou X, Wang X, Wang X: Prognosis for different patterns of distant metastases in patients with uterine cervical cancer: a population-based analysis. Journal of Cancer 2020, 11(6):1532-1541.

25. Yang L, Klint A, Lambe M, Bellocco R, Riman T, Bergfeldt K, Persson I, Weiderpass E: Predictors of ovarian cancer survival: a population-based prospective study in Sweden. International journal of cancer 2008, 123(3):672-679.

26. Greer JB, Modugno F, Ness RB, Allen GO: Anthropometry and the risk of epithelial ovarian cancer. Cancer 2006, 106(10):2247-2257.

27. Kim B, Park Y, Kim B, Ahn HJ, Lee KA, Chung JE, Han SW: Diagnostic performance of CA 125, HE4, and risk of Ovarian Malignancy Algorithm for ovarian cancer. Journal of clinical laboratory analysis 2019, 33(1):e22624.

28. Tarhriz V, Bandehpour M, Dastmalchi S, Ouladsahebmadarek E, Zarredar H, Eyvazi S: Overview of CD24 as a new molecular marker in ovarian cancer. Journal of cellular physiology 2019, 234(3):2134-2142.

29. Kim SI, Lee EJ, Lee M, Chung H, Kim JW, Park NH, Song YS, Kim HS: Recurrence patterns after bevacizumab in platinum-sensitive, recurrent epithelial ovarian cancer. International journal of gynecological cancer: official journal of the International Gynecological Cancer Society 2020, 30(12):1943-1950.

30. Tentes AA, Korakianitis OS, Kakolyris S, Kyziridis D, Veliovits D, Karagiozoglou C, Sgouridou E, Moustakas $\mathrm{K}$ : Cytoreductive surgery and perioperative intraperitoneal chemotherapy in recurrent ovarian cancer. Tumori 2010, 96(3):411-416.

31. Pokhriyal R, Hariprasad R, Kumar L, Hariprasad G: Chemotherapy Resistance in Advanced Ovarian Cancer Patients. Biomarkers in cancer 2019, 11:1179299x19860815.

32. Yue H, Wang J, Chen R, Hou X, Li J, Lu X: Gene signature characteristic of elevated stromal infiltration and activation is associated with increased risk of hematogenous and lymphatic metastasis in serous ovarian cancer. BMC cancer 2019, 19(1):1266.

33. Deng K, Yang C, Tan Q, Song W, Lu M, Zhao W, Lou G, Li Z, Li K, Hou Y: Sites of distant metastases and overall survival in ovarian cancer: A study of 1481 patients. Gynecologic oncology 2018, 150(3):460-465.

34. Guillon J, Petit C, Toutain B, Guette C, Lelièvre E, Coqueret O: Chemotherapy-induced senescence, an adaptive mechanism driving resistance and tumor heterogeneity. Cell cycle (Georgetown, Tex) 2019, 18(19):2385-2397.

\section{Tables}

Due to technical limitations, tables are only available as a download in the Supplemental Files section.

\section{Figures}




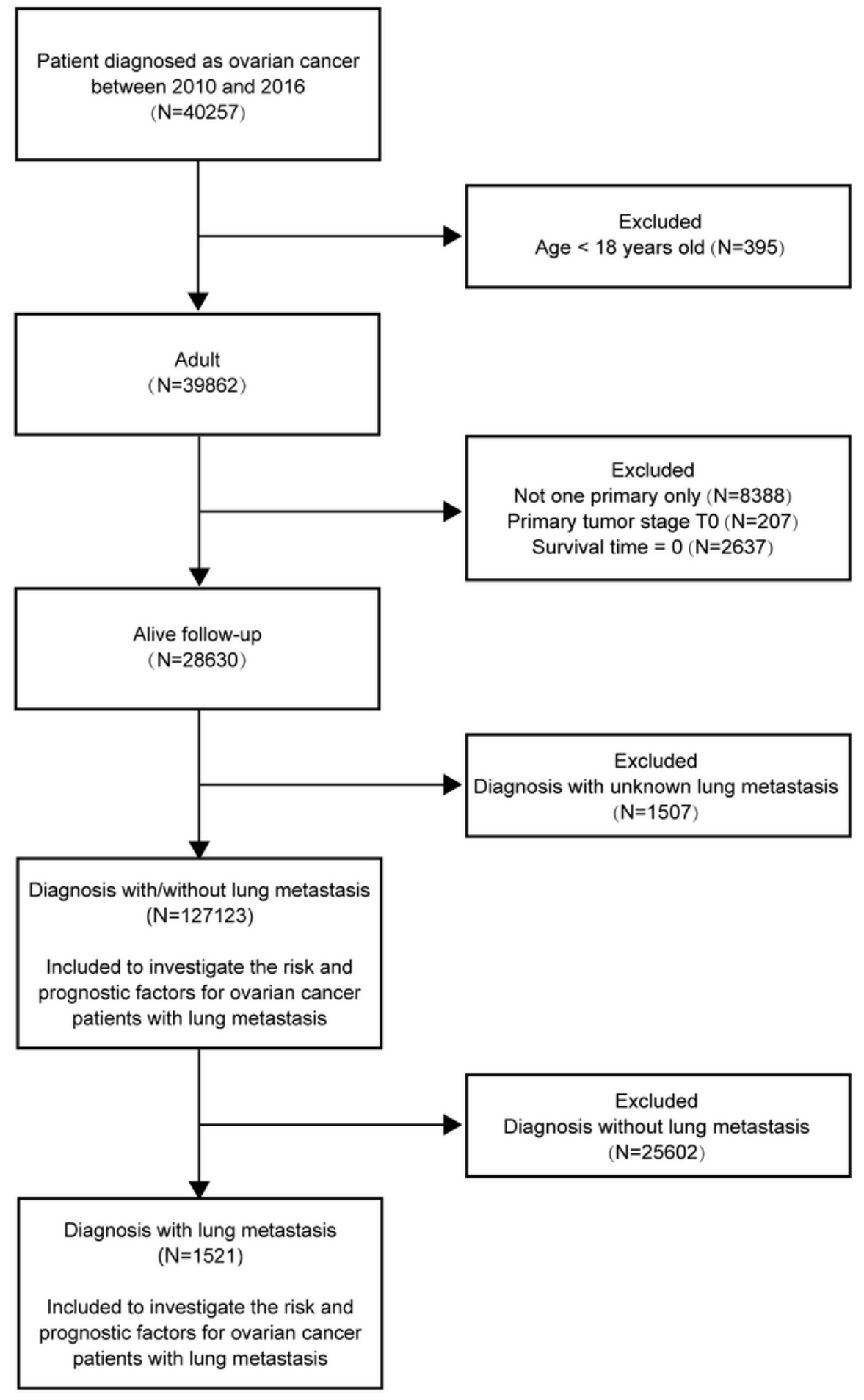

\section{Figure 1}

The flow diagram of participant inclusion and exclusion. 
A

Points
Marital status
Histology
Grade
Regional nodes examined
Radiotherapy
Chemotherapy
Surgical treatments on primary site
T stage
N stage
Bone metastasis
Brain metastasis
Liver metastasis
Total Points
Lung metastasis rate

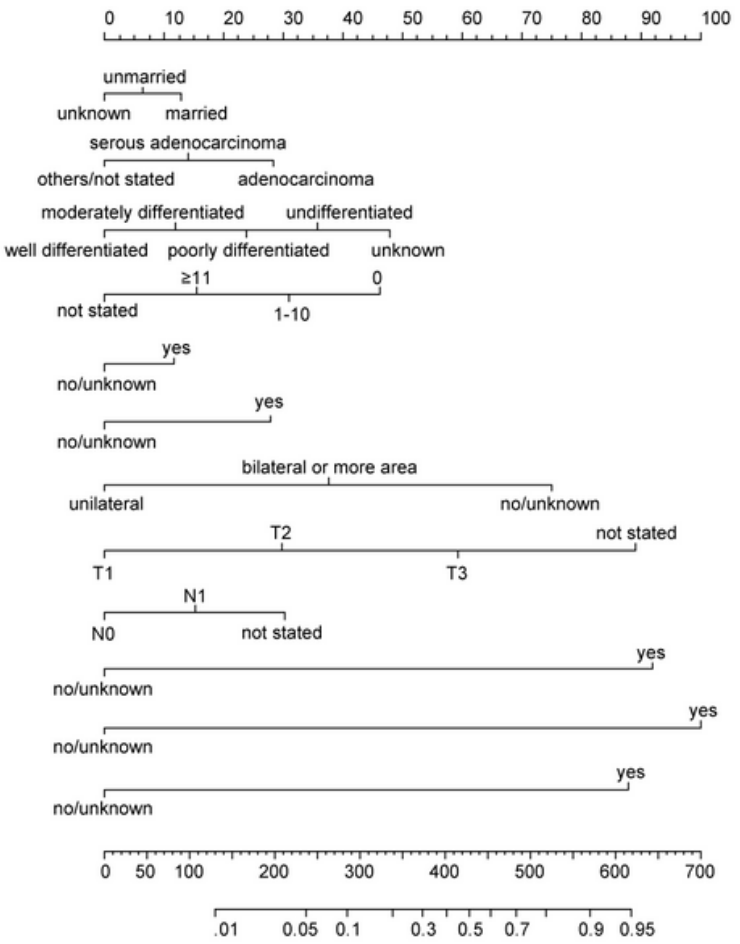

B

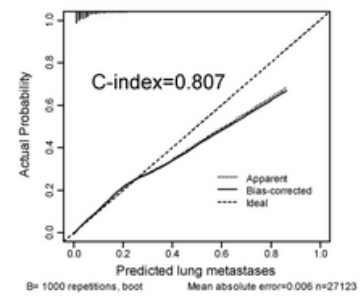

C

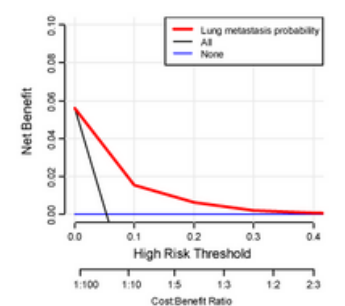

D

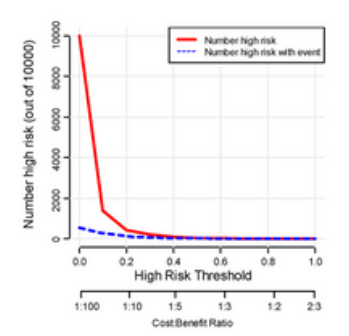

\section{Figure 2}

The nomogram combining with its calibration and verification curves for predicting LM morbidity in OC patients. A total of twelve factors were determined in LM incidence predictive nomogram (A). The calibration curve $(B)$ with the C-index of 0.807 was showed to verify the validity of prediction. Decision curve (C) and clinical impact curve (D) were plotted to show the event occurrence of patients with high risks. 

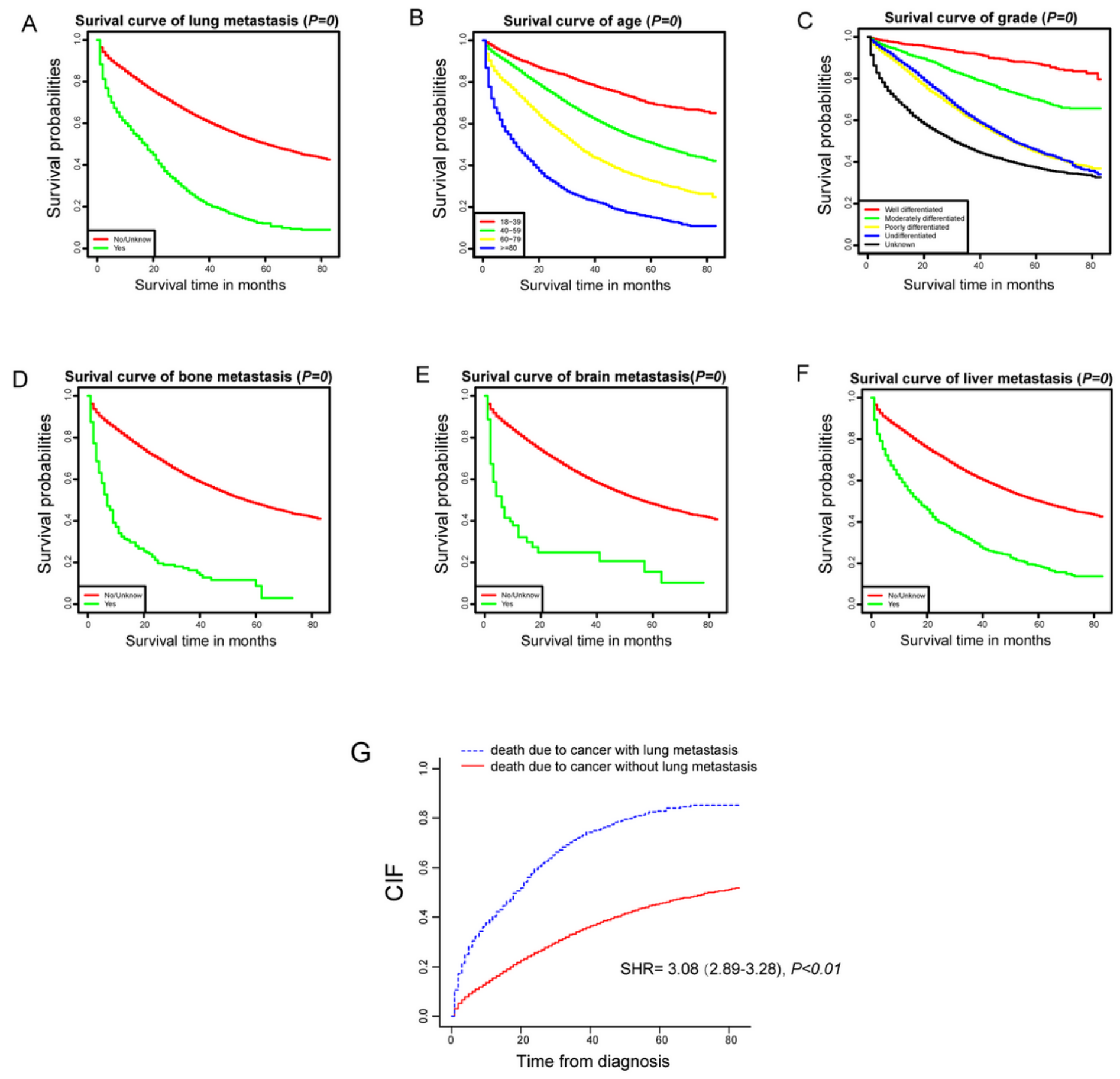

\section{Figure 3}

Kaplan-Meier analyses of OS among populations who were diagnosed with lung metastasis relative to the other $(A)$ and stratified by age $(B)$, grade $(C)$, bone metastasis $(D)$, brain metastasis $(E)$ and liver metastasis $(F)$ in ovarian carcinoma. And ovarian cancer-specific survival curve $(G)$ in ovarian carcinoma. 
A

Points
Age at diagnosis
Race
Marital status
Histology
Grade
Regional nodes examined
Radiotherapy
Chemotherapy
Surgical treatments on primary site
T stage
N stage
CA125
Bone metastasis
Brain metastasis
Liver metastasis
Total Points
3-Year survival
5-Year survival

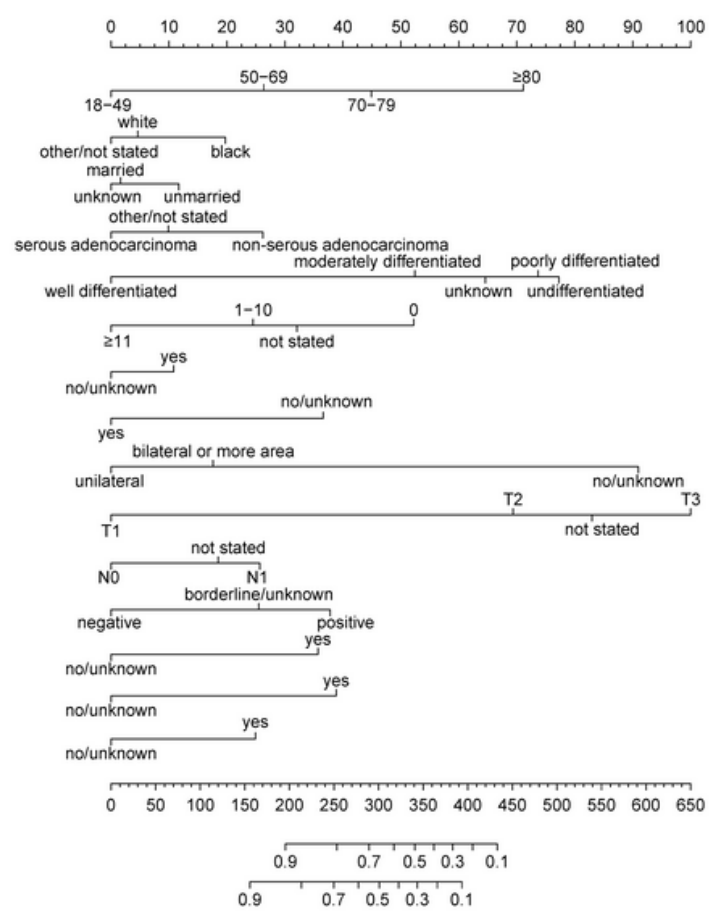

B
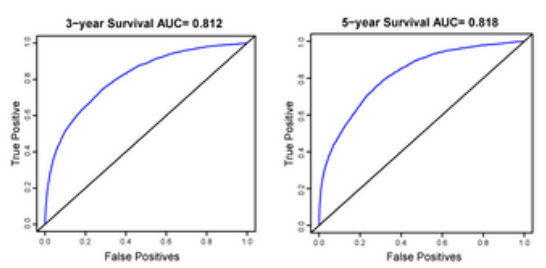

C
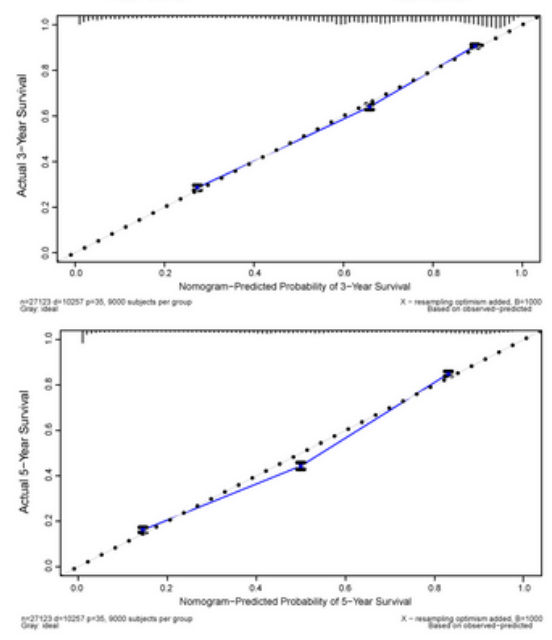

\section{Figure 4}

The nomogram combining with its calibration and verification curves for predicting risk factors on prognosis for $\mathrm{OC}$ patients. A total of fifteen prognostic factors were defined in 3- and 5-year survival nomogram (A). Calibration curves (B-C) with the values of AUC (3-year AUC $=0.812,5$-year AUC $=0.818$, respectively) were plotted to verify the effectiveness of prediction. 


\section{Clinic effect of OS nomogram}

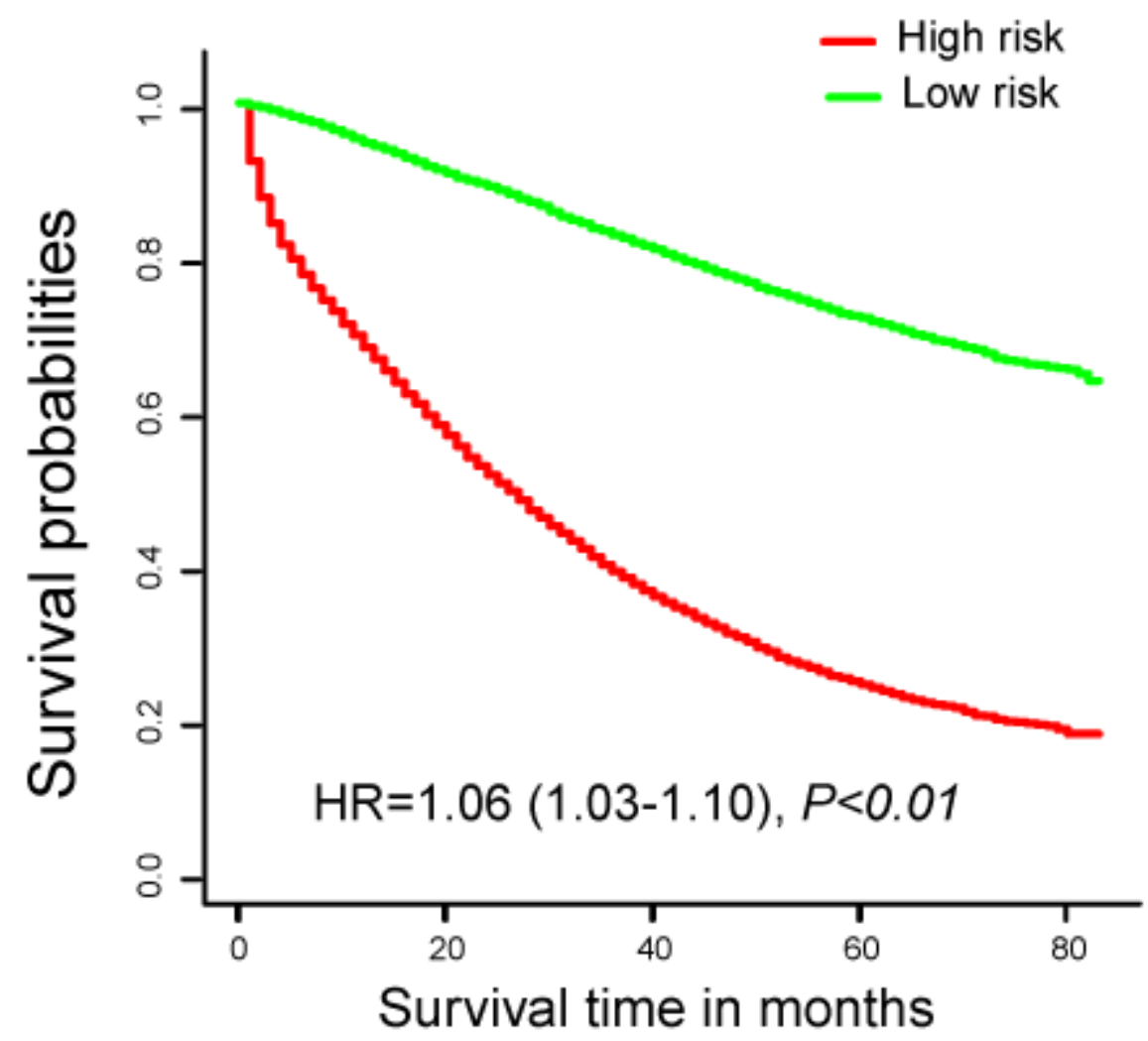

Figure 5

The clinical effect curve drawn from risk scores in the OS nomogram.

\section{Supplementary Files}

This is a list of supplementary files associated with this preprint. Click to download.

- Table1.tif

- Table2.tif

- Table3.tif 